BULLETIN OF THE

AMERICAN MATHEMATICAL, SOCIETY

Volume 77, Number 4, July 1971

\title{
$K$-THEORY OF A SPACE WITH COEFFICIENTS IN A (DISCRETE) RING
}

\author{
BY DAVID L. RECTOR ${ }^{1}$ \\ Communicated by M. L. Curtis, January 27, 1971
}

In [2], [3], S. Gersten has introduced higher $K$-groups of a ring which satisfy properties analogous to those of a generalized homology theory in a suitably defined homotopy category of rings [1]. In this announcement we use Gersten's $K$-groups to define for a ring $R$ a generalized cohomology theory $K_{R}^{*}()$, analogous to the AtiyahHirzebruch $K$-theory, on the category of finite simplicial sets so that $K_{R}^{*}(p t)=K_{\Sigma}^{*} R$, where $K_{\Sigma}^{*} R$ are Gersten's stable $K$-groups of the ring $R$. If $R$ is suitably restricted, in particular if it is commutative and regular, the theory $K_{R}^{*}($ ) will have products and Adams operations. One may also define, using the continuous theory in [6], a $K$-theory $K_{\Lambda}^{*}()$ with coefficients in a Banach ring $\Lambda$. This theory coincides with the Atiyah-Hirzebruch theory for $\Lambda=R, C$, or $H$. We give here an outline of proofs. A full account will appear elsewhere.

1. Definition of the theory. We recall the definition of Gersten's theory as given in [5]. Let $R$ be a ring (without unit). The functor $R \mapsto R[t]$ together with the natural transformations $R[t] \rightarrow R$ via " $t \rightarrow 1$ ", and $R[t] \rightarrow R\left[t, t^{\prime}\right]$ via $t \rightarrow t t^{\prime}$ define a cotriple in the category of rings. If $E R$ is the ideal $R[t] t$, then the restriction of those maps makes the functor $R \mapsto E R$ a cotriple. Associated to these cotriples are canonical simplicial rings $R[T]$ and $\bar{E} R$ with

$$
R[T]_{n}=R\left[t_{0}, \cdots, t_{n}\right], \quad \bar{E} R_{n}=E^{n+1} R .
$$

Let $Q R$ be the simplicial ring

$$
Q R=R[T] / \bar{E} R .
$$

One has

$$
K^{-i-1} R=\pi_{i} \text { Gl } Q R
$$

where $\mathrm{Gl}$ denotes the general linear group functor. This $K$-theory of rings is stabilized as follows [3]. Let $\Gamma R$ be the kernel of $R\left[t, t^{-1}\right]$ $\rightarrow R$. Then there is a natural homomorphism

AMS 1970 subject classifications. Primary 55B15, 55B20, 13D15, 16A54, $18 \mathrm{~F} 25$.

1 The author was partially supported by NSF grant GP-20552 during the preparation of this work.

Copyright (C) 1971, American Mathematical Society 


$$
K^{i} R \stackrel{\beta}{\rightarrow} K^{i-1} \Gamma R, \quad i \leqq 0,
$$

analogous to the Bott map, which is an isomorphism when $R$ is $K$-regular.

Put

$$
K_{\Sigma}^{i} R=\operatorname{inj} \lim _{n} K^{i-n} \Gamma^{n} R, \quad-\infty<i<\infty .
$$

Then if $R \rightarrow S \rightarrow T$ is a Gl-fibration [2], there is a long exact sequence

$$
\cdots \rightarrow K_{\Sigma}^{i} T \rightarrow K_{\Sigma}^{i} S \rightarrow K_{\Sigma}^{i} R \stackrel{\partial}{\rightarrow} K_{\Sigma}^{i+1} \rightarrow \cdots .
$$

Now to define a cohomology theory for simplicial sets, we will give a contravariant functor $(; R)$ from simplicial sets to rings which

(1) sends coproducts to products,

(2) sends cofibrations to Gl-fibrations,

(3) sends a point to $R$.

For $X$ and $Y$ simplicial sets, let $\Delta(X ; Y)$ denote the set of all simplicial maps from $X$ to $Y$. Put

$$
(X ; R)=\Delta(X ; Q R) .
$$

Then $(X ; R)$ is a ring and $(p t ; R)=R$, since $Q R_{0}=R$.

Definition 1.1. For $X$ a finite simplicial set, $K_{R}^{*}(X)=K_{\Sigma}^{*}(X ; R)$. The long exact sequence of a cofibration arises from

Proposition 1.2. If $Y \rightarrow X \rightarrow X / Y$ is a cofibration of simplicial sets then

$$
(X / Y ; R) \rightarrow(X ; R) \rightarrow(Y ; R)
$$

is a Gl-fibration.

This proposition follows from the following properties of the functor $\Delta(;)$.

LEMma 1.3. If $F$ is a functor which is left exact and preserves products then

$$
\Delta(X ; F Y)=F \Delta(X ; Y) \text {. }
$$

Proof. Follows from the fact that $F$ preserves equalizers.

LEMMA 1.4. If $Y$ is a simplicial object in a category with a forgetful functor to sets and $Y$ is contractible as a set complex then $\Delta(; Y)$ is an exact functor. 
To verify the homotopy axiom for the theory we must prove

Proposition 1.5. If $X \rightarrow Y$ is a map of finite simplicial sets which induces an isomorphism $H_{*}(X ; Z) \rightarrow H_{*}(Y ; Z)$, then $f^{l}: K_{R}^{*}(Y) \rightarrow K_{R}^{*}(X)$ is an isomorphism.

This proposition follows immediately from the fact that we have an analogue of the Atiyah-Hirzebruch spectral sequence defined intrinsically in the theory $K_{R}^{*}$ as follows.

Let $X^{n}$ be the $n$-skeleton of $X$. We have a tower of Gl-fibrations

$$
\cdots \rightarrow\left(X^{n} ; R\right) \rightarrow\left(X^{n-1} ; R\right) \rightarrow \cdots \rightarrow\left(X^{0} ; R\right) .
$$

The long exact $K_{\Sigma}^{*}$-theory sequences of these fibrations define a homology exact couple. The spectral sequence of that couple converges strongly to $K_{R}^{*}(X)$. One has $E_{1}^{p, q}=K_{\Sigma}^{p+q}\left(X^{p} / X^{p-1} ; R\right)$. By a brute force calculation

Lemma 1.6. $K_{\Sigma}^{p+q}\left(X^{p} / X^{p-1} ; R\right)=\oplus_{\sigma} K_{\Sigma}^{p+q} R$, where the sum runs over all nondegenerate $p$-simplexes $\sigma$ of $X$.

Standard diagram chases now establish

THEOREM 1.7. There is a natural spectral sequence $\left\{E_{r}\right\}$ converging to $K_{R}^{*}(X)$ with

$$
E_{2}^{p, q}=H^{p}\left(X ; K_{\Sigma}^{q} R\right)
$$

Thus

THEOREM 1.8. $K_{R}^{*}()$ is a generalized cohomology theory on the category of finite simplicial sets.

In addition,

THEOREM 1.9. $K_{R}^{*}(\mathrm{l})$ depends only on the ring homotopy type of $R$ and if $R \rightarrow S \rightarrow T$ is a Gl-fibration of rings there is a natural exact triangle of theories

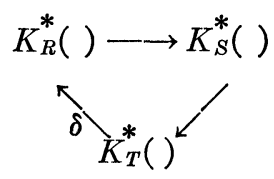

where $\delta$ has degree +1 .

2. Products and Adams operations. Let $R$ and $T$ be rings, $X$ and $Y$ simplicial sets. We then have a pairing 


$$
(X ; R) \otimes z(Y ; T) \stackrel{\phi}{\rightarrow}(X \times Y ; R \otimes T)
$$

given by

$$
\phi(\alpha \otimes \beta)(x, y)=\alpha(x) \otimes \beta(y) .
$$

Using the product structure in $K_{\Sigma}^{*}()$ [4] one has

THEOREM 2.1. There is a natural graded associative pairing

$$
K_{R}^{*}(X) \otimes K_{T}^{*}(Y) \rightarrow K_{R \otimes T}^{*}(X \times Y) .
$$

If $R$ is a commutative ring there is a natural graded commutative ring structure on $K_{R}^{*}(X)$ arising from the diagonal $\Delta: X \rightarrow X \times X$.

Now suppose that $R$ is a $K$-regular ring [2]. From a truncated version of the spectral sequence of Theorem 1.7 one has

Theorem 2.2. If $R$ is $K$-regular,

$$
K_{\Sigma}^{i}(X ; R)=K^{i}(X ; R)
$$

for $i \leqq 0$.

Now the theory $K^{i}$ has Adams operations which are graded ring homomorphisms. Let $K_{R}^{-}(\mathrm{l})$ be the nonpositive graded part of $K_{R}^{*}(\mathrm{)}$. Then

THEOREM 2.3. If $R$ is $K$-regular there are natural graded ring morphisms

$$
\psi^{k}: \overline{K_{R}}(X) \rightarrow \overline{K_{R}}(X)
$$

for $k \geqq 0$. The $\psi^{k}$ commute with the boundary of the long exact sequence of a cofibration when that makes sense.

REMARK 2.4. Using the continuous polynomials of [6] one may define a theory $K_{\Lambda}^{*}(\mathrm{)})$ for $\Lambda$ a valuation ring. For $\Lambda=R, C$ or $H$ there is a natural equivalence

$$
K_{\Delta}^{*}() \rightarrow K \Lambda^{*}()
$$

where $K \Lambda^{*}$ is the $K$-theory of Atiyah and Hirzebruch. It would be interesting to know the coefficient group $K_{\Lambda}^{*}(p t)=K^{*} \Lambda$ for $\Lambda=Q_{p}$ or $\boldsymbol{Z}_{p}$.

REMARK 2.5. The ring complex $Q R$ above may be replaced by the nicer ring complex $\Delta R$ where

$$
\Delta R_{n}=R\left[t_{0}, \cdots, t_{n}\right] / t_{0}+\cdots+t_{n}-1,
$$


and

$$
\begin{array}{rlrl}
d_{i} t_{j} & =t_{j}, & & i>j, \\
& =0, & & i=j, \\
& =t_{j-1}, & & i<j, \\
s_{i} t_{j} & =t_{j}, & & i>j, \\
& =t_{j}+t_{j+1}, & i=j, \\
& =t_{j+1}, & & i<j .
\end{array}
$$

One may now redefine $(X ; R)$ as the ring of simplicial maps of $X$ to $\Delta R$. The same $K$-theory for $X$ now arises in view of

Proposition 2.6. $\pi_{i}$ Gl $\Delta R=K^{-i-1} R, i \geqq 0$.

This proposition is proved by showing that $\pi_{i}$ Gl $\Delta R$ satisfies the axioms for $K^{-i-1} R[2]$.

\section{REFERENCES}

1. S. M. Gersten, Homotopy theory of rings (to appear).

2. - On Mayer-Vietoris functors and algebraic K-theory, J. Algebra (to appear).

3. - Stable K-theory of discrete rings (to appear).

4. - - Stable K-theory of discrete rings. II: Product and transfer (to appear).

5. S. M. Gersten and D. L. Rector, A relation between two simplicial algebraic $K$ theories, Bull. Amer. Math. Soc. 77 (1971), 397-399.

6. M. Karoubi and O. Villamayor, Foncteurs $K^{n}$ en algèbre et en topologie, C. R. Acad. Sci. Paris Sér. A-B 269 (1969), A416-A419. MR 40 \#4944.

Rice University, Houston, Texas 77001 